\title{
Meaningful Use of Electronic Prescribing in 5 Exemplar Primary Care Practices
}

Jesse C. Crosson, $P b D^{1}$

Rebecca S. Etz, $P b D^{1}$

Shinyi $W u, P b D^{2,3}$

Susan G. Straus, $P b D^{4}$

David Eisenman, MD, MSHS $S^{3,5}$

Douglas S. Bell, MD, PbD ${ }^{3,5}$

'Research Division, Department of Family Medicine and Community Health, UMDNJ-Robert Wood Johnson Medical School, Somerset, New Jersey

${ }^{2}$ Epstein Department of Industrial and Systems Engineering, University of Southern California, Los Angeles, California

${ }^{3}$ RAND Corporation, Los Angeles, California

${ }^{4}$ RAND Corporation, Pittsburgh, Pennsylvania

${ }^{5}$ Division of General Internal Medicine and Health Services Research, David Geffen School of Medicine, University of California at Los Angeles, Los Angeles, California

Conflicts of interest: authors report none.

\section{CORRESPONDING AUTHOR}

Jesse C. Crosson, $\mathrm{PhD}$

Research Division

Department of Family Medicine

and Community Health

UMDNJ-Robert Wood Johnson

Medical School

1 World's Fair Dr, Room 1500

Somerset, NJ 08873

jesse.crosson@umdnj.edu

\begin{abstract}
PURPOSE Successful use of electronic prescribing (e-prescribing) is a key requirement for demonstrating meaningful use of electronic health records to qualify for federal incentives. Currently, many physicians who implement e-prescribing fail to make substantial use of these systems, and little is known about factors contributing to successful e-prescribing use. The objective of this study was to identify successful implementation and use techniques.
\end{abstract}

METHODS We conducted a multimethod qualitative case study of 5 ambulatory primary care practices identified as exemplars of effective e-prescribing. The practices were identified by a group of e-prescribing experts. Field researchers conducted in-depth interviews and observed prescription-related workflow in these practices.

RESULTS In these exemplar practices, successful use of e-prescribing required practice transformation. Practice members reported extensive efforts to redesign work processes to take advantage of e-prescribing capabilities and to create specific e-prescribing protocols to distribute prescription-related work among practice team members. These practices had substantial resources to support e-prescribing use, including local physician champions, ongoing training for practice members, and continuous on-site technical support. Practices faced considerable challenges during use of e-prescribing, however, deriving from problems coordinating new work processes with pharmacies and ineffective health information exchange that required workarounds to ensure the completeness of patient medical records.

CONCLUSIONS More widespread implementation and effective use of e-prescribing in ambulatory care settings will require practice transformation efforts that focus on work process redesign while being attentive to effects on patient and pharmacy involvement in prescribing. Improved health information exchange is required to fully realize expected quality, safety, and efficiency gains of e-prescribing.

Ann Fam Med 2011;9:392-397. doi:10.1370/afm.1261.

\section{INTRODUCTION}

$\mathrm{P}$ rescription drugs are among the most commonly used treatments in primary care, and the prescribing process has been a central target of primary care transformation efforts. Early efforts to transform prescribing focused on electronic prescribing (e-prescribing) as an initial step that could be achievable for most practices. ${ }^{1}$ Although 234,000 US physicians have adopted e-prescribing over the last decade, many adopters revert to handwriting for most of their prescriptions. ${ }^{2-4}$ The American Recovery and Reinvestment Act of 2009 (ARRA) created a new program to promote the "meaningful use" of more-comprehensive electronic health record (EHR) systems. ${ }^{5}$ The meaningful use criteria include requirements that prescribers transmit $40 \%$ of permissible prescriptions electronically using certified EHR technology in order to qualify for incentive payments. ${ }^{6}$ Although incentives are likely to spur adoption of e-prescribing, experience to date suggests that a majority are likely to fall short of the meaningful use mark. ${ }^{7}$ New approaches to e-prescribing adoption are thus 
needed if federal policy goals are to be achieved and practices are to qualify for incentive payments.

Previous work has documented some of the challenges faced by those adopting e-prescribing in primary care settings, including unrealistic expectations among users, inadequate technical support, and poor functioning of the technical infrastructure for e-prescribing. ${ }^{8,9}$ These challenges have slowed adoption while leading some early adopters to either abandon e-prescribing or delegate its use to support staff, thereby neutralizing its potential to influence prescribing decisions. ${ }^{8,10}$ Nonetheless, some e-prescribers have successfully transformed their practices to make use of the technology, and lessons from these successes can inform wider adoption of e-prescribing.

We present findings from a study of practices that have successfully adopted and meaningfully used e-prescribing to identify key lessons for primary care transformation efforts.

\section{METHODS}

We conducted a comparative case study among 5 exemplar e-prescribing practices. Successful strategies were identified at each site and then compared, across sites, to identify generalizable lessons. Exemplar sites were identified in collaboration with an Expert Advisory Committee (EAC), composed of executives whose organizations had been involved with e-prescribing implementation initiatives (Supplemental Table 1, avail-

able at http://www.annfammed.org/cgi/content/ full/9/5/392/DC1). First, EAC members identified practices that submitted a high volume of their prescriptions electronically and had innovative implementation processes. Second, we purposively sampled from that group to ensure inclusion of safety net settings, practices of varying size, practices that had implemented e-prescribing in the context of a full EHR adoption, and those that implemented e-prescribing as a stand-alone application. An advance questionnaire was used to ensure that practices met the criteria of our purposive sample (Supplemental Appendix, avail-

able at http://www.annfammed.org/cgi/content/ full/9/5/392/DC1).

From March to May 2009, we conducted 3-day site visits at each practice that included in-depth interviews with practice members and observations of practice culture and prescribing processes. During site visits, we checked observations with practice members to ensure accuracy of field notes. Interview transcripts, field notes, and responses to the advance questionnaire were entered into ATLAS.ti software (ATLAS. ti Scientific Software Development GmbH, Berlin, Germany) for coding and analysis by a multidisci- plinary team using a template organizing style. ${ }^{11}$ Coding proceeded in a 3 -stage process. We used an initial codebook to code data from 1 practice and to develop a shared understanding of code definitions and use. Next, we coded data from a second practice independently, and discrepancies were resolved through group consensus, refining code definitions where necessary. The remaining data were coded independently by 2 coauthors (J.C.C., R.S.E.), and discrepancies were resolved by consensus. Coding reports were generated and analyzed using a grounded theory approach to identify common themes and to select representative text segments. ${ }^{12}$

Institutional review boards at UMDNJ-Robert Wood Johnson Medical School, RAND Corporation, and University of California at Los Angeles approved the study.

\section{RESULTS}

All sites were ambulatory primary care practices of varying size and organizational affiliation (Table 1). Three sites used an e-prescribing system that was integrated with an EHR, whereas 2 sites used a standalone e-prescribing system. All sites had substantial on-site information technology (IT) resources to supplement vendor-provided services. Meaningful use of e-prescribing in these practices proceeded through 3 phases: planning, implementation, and use. Best practices for each of these phases are described below and summarized in Table 2. As indicated in Table 2 and in the descriptions below, many of the strategies associated with successful uptake of e-prescribing were important across phases.

\section{Planning}

Technical support and planning teams worked with sites before implementation to understand existing work processes and how these could be affected by e-prescribing. The value of this work was often not appreciated until after implementation:

We spent tons and tons of time, initially before we got the system...went through every step of everything we did...I didn't quite get... why we were spending so much time. But now I see that's what made it easier. ... Every step of everything every person does in the office had to be transformed.

Some practice members understood from the outset that using e-prescribing would require a shift in work processes:

It's not doing the same thing you've always done but now doing it electronically. It's different. It's a ton of workflow changes. You have to change the way you do business.... You're going to do a different job now. 
Table 1. Characteristics of Exemplar Electronic Prescribing Practices

\begin{tabular}{|c|c|c|c|c|c|c|c|}
\hline $\begin{array}{l}\text { Practice No. } \\
\text { and Structure }\end{array}$ & Type & Records & $\begin{array}{l}\text { Nature } \\
\text { of eRx }\end{array}$ & $\begin{array}{l}\text { No. of } \\
\text { eRx Users }\end{array}$ & $\begin{array}{l}\text { Source of } \\
\text { IT Support }\end{array}$ & $\begin{array}{l}\text { No. of } \\
\text { Rx/Month }\end{array}$ & $\begin{array}{c}\text { No. of } \\
\text { Visits/Year }\end{array}$ \\
\hline 1. Part of an IPA & $\begin{array}{l}\text { Internal medicine, } \\
\text { pediatrics }\end{array}$ & Paper & Stand-alone & $\begin{array}{l}4 \text { at site/1 CMA } \\
\text { responsible }\end{array}$ & $\begin{array}{l}\text { IPA (through } \\
\text { contractor) }\end{array}$ & 1,600 & 12,000 \\
\hline $\begin{array}{l}\text { 2. Part of medi- } \\
\text { cal group }\end{array}$ & $\begin{array}{l}\text { Multiple-specialty } \\
\text { practice }\end{array}$ & EHR & Integrated & $\begin{array}{l}25 \text { at site/2 RNs } \\
\text { responsible }\end{array}$ & $\begin{array}{l}\text { Internal } \\
\text { department }\end{array}$ & 9,839 & 112,000 \\
\hline $\begin{array}{l}\text { 3. Associated } \\
\text { with hospital }\end{array}$ & Pediatrics & $\begin{array}{l}\text { Hybrid paper } \\
\text { and EHR }\end{array}$ & Integrated & $\begin{array}{l}8 \text { at site } / 4 \text { RNs } \\
\text { responsible }\end{array}$ & Hospital & 1,176 & 22,468 \\
\hline $\begin{array}{l}\text { 4. Associated } \\
\text { with hospital }\end{array}$ & $\begin{array}{l}\text { Internal and fam- } \\
\text { ily medicine }\end{array}$ & EHR & Stand-alone & $\begin{array}{l}10 \text { at site/3 RNs } \\
\text { responsible }\end{array}$ & Hospital & 8,000 & 25,000 \\
\hline $\begin{array}{l}\text { 5. Part of medi- } \\
\text { cal group }\end{array}$ & $\begin{array}{l}\text { Multiple-specialty } \\
\text { practice }\end{array}$ & EHR & Integrated & $\begin{array}{l}60 \text { at site } / 5 \text { phar- } \\
\text { macy techni- } \\
\text { cians dedicated }\end{array}$ & $\begin{array}{l}\text { Internal } \\
\text { department }\end{array}$ & - & - \\
\hline
\end{tabular}

$\mathrm{CMA}=$ certified medical assistant; $\mathrm{EHR}=$ electronic health record; $\mathrm{eRx}=$ electronic prescribing; IPA = independent physicians association; IT = information technology; $\mathrm{RN}=$ registered nurse; $\mathrm{Rx}=$ prescription.

Note: Prescribers are physicians, physician assistants, or nurse practitioners. Sources were advance questionnaires, and interviews conducted by and observations made by coauthors.

a Estimates provided by practice leaders; leaders of practice 5 did not provide estimates.

Workflow redesign among exemplar sites typically included standardizing previously idiosyncratic documentation patterns. Planning teams developed protocols that allowed for standardized handling of prescription renewals by nonphysician practice members. At one site, such protocols served as the basis for a care management system focused on ensuring reliable follow-up for patients with chronic conditions. Once the practices implemented e-prescribing, staff with newly assigned prescribing tasks expressed increased

\section{Table 2. Best Practices at Each Phase of Electronic Prescribing Adoption}

\begin{tabular}{ll}
\hline Phase & Best Practices \\
\hline Planning & Identify organizational champion and/or sup- \\
portive leader \\
Examine existing work processes to determine \\
how these might be affected by implementation \\
Plan workflow and work process changes, stan- \\
dardize data entry processes, and develop \\
protocols \\
Introduce pharmacists and patients to new \\
processes \\
Provide comprehensive, on-site training pro- \\
gram focused on communicating benefits of \\
e-prescribing to practice members, test runs \\
with software, and introduction to new work \\
processes, standards, and protocols \\
Identify and train super users \\
Ensure availability of ongoing technical support \\
Assess accuracy of formulary information \\
(through communication with pharmacies) \\
Monitor pharmacy and patient compliance with \\
new work processes \\
Ensure completeness of medication history infor- \\
mation in the medical record through work- \\
arounds for paper prescriptions \\
Analyze and modify work processes as needed \\
Ensure availability of ongoing technical support
\end{tabular}

job satisfaction and a greater sense of professionalization. One nurse expressed appreciation for the expansion of her responsibilities: "I have skills, and I can do more. So, then they got a protocol, which is absolutely wonderful." Another site developed a centralized office in which pharmacy technicians handled renewals and reported "the technicians feel empowered and professionalized, and the doctors feel like they have been able to return to what doctors are supposed to do... they make decisions."

Implementation planning among exemplars often included formal communications with pharmacists and patients to introduce the new e-prescribing processes. Some practices developed a written explanation for how e-prescribing affected common work processes and referred to this when interacting with patients and pharmacies. One practice provided prescriptionsized handouts for patients explaining the benefits of e-prescribing to the patient on one side and providing information to pharmacists regarding where to find electronically transmitted prescriptions on the other (Supplemental Figure 1, available online at http:// www.annfammed.org/cgi/content/full/9/5/392).

Three practices had an identifiable and respected physician champion who played key planning roles and articulated a clear vision for use of e-prescribing technology. Champions enhanced implementation success through their understanding of practice needs, their problem-solving skills, and their roles as opinion leaders. In the 2 sites without a clear physician champion, support from practice leaders for e-prescribing was essential. At both of these sites, the decision to e-prescribe was seen as a good business decision and was supported by the larger medical group, which then in turn provided the necessary plan- 
ning and technical resources to ensure success. Physician champions continued to serve important roles during implementation and use.

\section{Implementation}

Four sites had technical support departments focused on training and implementation. In the remaining site, similar support was provided by an outside organization through a contractual arrangement. In all cases, technical support included providing real-time solutions for problems encountered during implementation, such as adjusting automated drug-drug interaction alerts to better match system sensitivity with the user's expertise. Technical support was also essential for helping practices create strategies to use medication lists and drug dictionaries effectively. All practices had ongoing access to technical expertise throughout the implementation phase. This IT expertise included both hardware and software support. In sites affiliated with larger organizations, practices had access to "help desk" telephone-based assistance in addition to regular on-site visits from technical support staff.

Implementation at each practice included comprehensive training and education strategies that communicated the rationale for e-prescribing and built familiarity with key system features. Formalized education for practice members emphasized the benefits of e-prescribing and focused on setting realistic expectations about technology capabilities. Training often included hands-on exercises and access to the software before implementation. Practices instructed members regarding new work processes, such as standards for where free texting (entry of text in the user's own words) was permitted and where it should be avoided in the e-prescription process.

Practices typically designated "super users," who received more extensive training than other members of the health care team and were then given greater access to program features and settings than regular users. Super users were thus able to assist with implementation by quickly addressing frustrations of new users, troubleshooting problems, and helping prevent interruptions in workflow that might lead to rejection of the technology. Specific tasks of super users included "locating a template, setting up preferences, ...printers not working...just little things like that." Some practice champions were also super users. One champion/super user set up templates for commonly used prescriptions enabling users to click on appropriate prescription instructions rather than typing them in each time. Super users thus played a key bridging role between the clinical staff and the technical support staff. This role was seen as essential to successful implementation.

\section{Use}

After e-prescribing implementation, exemplar sites continued to actively address challenges and difficulties that they encountered. At one practice, a user group was formed to share strategies for finding particular medications in the system and for customizing user preferences. At another practice, physicians worked together with physician champions or super users to create multiple "favorites" lists customized for specific patient types.

Nonetheless, the practices experienced ongoing challenges with use of e-prescribing. In all sites, e-prescribers continued to report low levels of confidence in formulary, benefit, and medication history data present in their systems and derived through information exchange. Even though certified e-prescribing systems are generally capable of conducting transactions to check patients' insurance coverage, download medication history based on prescription drug claims, and match prescriptions to formulary and benefit information for the patients' plan, all practices faced challenges with using these data. Reported one physician:

(It's) not that good in our system because...(what we get is) not necessarily...(the) formulary...(from) the health plan.

..I've pretty much learned on my own which health plan favors what...(but) I would love to see (the system) really reflect what's the true lower copay for a patient.

This lack of confidence in formulary data limited the potential for using e-prescribing to offer more cost-effective prescriptions to patients. Prescribers also expressed a lack of confidence in the completeness of patient-specific data presented in e-prescribing systems, and many developed parallel systems for collecting and maintaining medication history data, thereby limiting potential work efficiencies.

Another problem faced by these sites was the receipt of prescription renewal requests via fax, even when the original prescription had been transmitted electronically. Such receipt required sites to manually enter faxed renewal requests into their system to maintain record integrity. Although practices attempted to communicate with pharmacies and patients regarding e-renewal processes, practice members reported that pharmacies often lacked an awareness or understanding of the problem. One office manager said:

You get (faxed renewals from pharmacies) and the fax says, 'Bogged down with paper? Use our electronic refills!' So, we had someone calling them and saying why did you send this by fax?... They'd say 'We don't fax.'...(Actually) it's automated...(and) they have no clue how their systems work.

This problem continued despite efforts to register prescribers as accepting e-refill requests through the 
Surescripts routing network. Some practice members reported that pharmacies preferred to trust their own systems regarding prescriber and practice contact information rather than rely on the Surescripts database. Doing so led to data discrepancies that then converted e-prescriptions to faxed prescriptions.

Recent Medicaid program changes requiring the use of security forms with wet signatures (handwritten signatures) for certain medications created additional challenges for these practices because these prescriptions had to be manually recorded to ensure the completeness of patient records:

We have to print them out and fax them to the pharmacy.. (since) it has to be a wet signature.... We've taken 10 steps backwards for all the work we've done to get e-prescribing going.

Some sites also reported difficulties sending e-prescriptions to mail order pharmacies or Veterans Administration pharmacies. In each of these sites, these technical challenges required the development of workarounds. Access to technical support from IT support departments, either within the practice or available as needed, and experience with work process redesign developed throughout the planning and implementation phases were essential for ensuring that these difficulties did not lead to abandonment of e-prescribing.

\section{DISCUSSION}

E-prescribing features prominently among the technologies that are expected to transform health care in the near future, offering the potential to increase medication safety through the use of automated alerts delivered at the point of care, reduce costs through greater use of generics and lower-cost medications, and reduce call-backs due to poor handwriting. ${ }^{13,14}$ The planning, implementation, and use strategies followed by exemplary sites suggest some factors that may enable a broader range of practices to achieve meaningful use. We found that successful adoption of e-prescribing required substantial investments of planning time and the ongoing transformation of work processes. Specifically, it required that practices (1) have at least 1 practice member able to articulate the importance of e-prescribing within the process of clinical care delivery; (2) develop communication strategies for introducing and orienting patients and pharmacies to e-prescribing processes $_{i}(3)$ have access to technical support throughout the implementation process capable of responding to problems before user frustration impeded adoption success; and (4) focus on transforming work processes to effectively incorporate e-prescribing.
Careful planning and implementation of redesigned work processes was a common element among all exemplar sites. In particular, all practices had implemented protocols allowing office staff to assist in handling prescription renewal requests, thus helping to offset the extra time that e-prescribing often takes for prescribers to generate prescriptions. ${ }^{15}$ In addition, we found that the staff in exemplar practices typically welcomed the opportunity to expand their job responsibilities.

The Health Information Technology for Economic and Clinical Health Act (HITECH) provisions of ARRA have established health IT regional extension centers (RECs) intended to provide practices with technology implementation assistance. ${ }^{16}$ Our findings suggest that the RECs will serve an important role in fostering adoption of health IT in that all of the successful practices we observed had strong external assistance for making the transition to e-prescribing. Key elements of this assistance included technical support for setup and troubleshooting for hardware, software, and network connections. At 4 sites, this support was delivered by internal IT staff, while the 5 th site successfully used their independent physician association to contract for this support, an approach that is more likely to be followed by the majority of smaller practices in the United States.

Furthermore, technical support appeared to work best when support personnel were readily available to visit practices for setup, training, and troubleshooting. By contrast, technical support in sites that struggled or failed with e-prescribing in an earlier study appeared to be less accessible, spend less time in the practice near the launch time, exhibit longer lags in response to support calls, and deliver more support by telephone rather than in person. ${ }^{8}$ These findings regarding appropriate levels of technical support should be considered when determining the extent of services offered by the RECs.

The qualitative methods that we used to examine these practices helped us to identify factors associated with successful implementation and use of e-prescribing in these sites; however, our study is limited in that these methods do not allow us to determine the relative importance of individual factors. An assessment of their relative importance would require a larger observational study, and the findings from such a study would be of obvious benefit to primary care practice leaders as they work toward more meaningful use of this technology. In addition, because we did not interview patients in this study, we were unable to directly determine what role they played in the implementation process.

E-prescribing is seen as one way to reduce health care costs by helping prescribers and patients select 
effective but lower-cost medications. ${ }^{17,18}$ Reducing patient costs could address a common barrier to adherence to medication therapies. ${ }^{19,20}$ To realize these aims, however, our study suggests that better functioning health information exchange for e-prescribing is needed. The accuracy and completeness of individual patient formularies and medication histories presented and exchanged in e-prescribing systems still falls short of prescribers' expectations. Providing such information reliably will be essential if e-prescribing is to contribute effectively to the improved safety, quality, and cost-effectiveness of health care.

The HITECH provisions of ARRA are designed to encourage widespread EHR and e-prescribing use after a large, but relatively short-term, investment. Our results suggest that e-prescribing success, and likely that of other health ITs, will require a longer-term commitment to supporting practice transformation, sufficient resources to aid in making these changes, and improvements to the infrastructure for health information exchange.

To read or post commentaries in response to this article, see it online at http://www.annfammed.org/cgi/content/full/9/5/392.

Key words: Electronic health records; electronic prescribing; information technology; physicians' practice patterns; practice-based research; primary care; workflow

Submitted August 27, 2010; submitted, revised, February 7, 2011; accepted March 2, 2011.

Funding support: This study was funded in part through grant 1 U18 HS016391-01 and through contract HHSA290200600017, TASK ORDER \#4, both from the US Agency for Healthcare Research and Quality.

Disclaimer: The opinions expressed in this document are those of the authors and do not reflect the official position of AHRQ or the US Department of Health and Human Services.

Acknowledgments: We are grateful for the assistance of Christopher Jackson and Karen Danna-Lynch in developing data-coding schemes.

\section{References}

1. Bell DS, Friedman MA. E-prescribing and the Medicare Modernization Act of 2003. Health Aff (Millwood). 2005;24(5):1159-1169.

2. Surescripts. National progress report on e-prescribing. 2010. http:// www.surescripts.com/about-e-prescribing/progress-reports.aspx. Accessed August 23, 2011.
3. Fischer MA, Vogeli C, Stedman M, Ferris T, Brookhart MA, Weissman JS. Effect of electronic prescribing with formulary decision support on medication use and cost. Arch Intern Med. 2008;168(22): 2433-2439.

4. Pevnick JM, Asch SM, Adams JL, et al. Adoption and use of standalone electronic prescribing in a health plan-sponsored initiative. Am J Manag Care. 2010;16(3):182-189.

5. Blumenthal D. Stimulating the adoption of health information technology. N Engl J Med. 2009;360(15):1477-1479.

6. Blumenthal $D$, Tavenner $M$. The "meaningful use" regulation for electronic health records. N Engl J Med. 2010;363(6):501-504.

7. Blumenthal D. Launching HITECH. N Engl J Med. 2010;362(5): 382-385.

8. Crosson JC, Isaacson N, Lancaster D, et al. Variation in electronic prescribing implementation among twelve ambulatory practices. J Gen Intern Med. 2008;23(4):364-371.

9. Bell DS, Schueth AJ, Guinan JP, Wu S, Crosson JC. Evaluating the technical adequacy of electronic prescribing standards: results of an expert panel process. AMIA Annual Symposium Proceedings/AMIA Symposium AMIA Symposium. 2008;Jan 1:46-50.

10. Grossman JM, Gerland A, Reed MC, Fahlman C. Physicians' experiences using commercial e-prescribing systems. Health Aff (Millwood). 2007;26(3):w393-w404.

11. Crabtree BF, Miller WL. Using codes and code manuals: a template organizing style of interpretation. In: Crabtree BF, Miller WL, eds. Doing Qualitative Research. 2nd ed. Thousand Oaks, CA: Sage Publications; 1999:163-177.

12. Corbin JM, Strauss AL. Basics of Qualitative Research: Techniques and Procedures for Developing Grounded Theory. 3rd ed. Los Angeles, CA: Sage Publications, Inc; 2008.

13. Institute of Medicine. Committee on Quality of Health Care in America. Crossing the Quality Chasm: A New Health System for the 21st Century. Washington, DC: National Academy Press; 2001.

14. Kohn LT, Corrigan J, Donaldson MS. To Err Is Human: Building a Safer Health System. Washington, DC: National Academy Press; 2000.

15. Hollingworth W, Devine EB, Hansen RN, et al. The impact of e-prescribing on prescriber and staff time in ambulatory care clinics: a time motion study. J Am Med Inform Assoc. 2007;14(6):722-730.

16. Maxson E, Jain S, Kendall M, Mostashari F, Blumenthal D. The regional extension center program: helping physicians meaningfully use health information technology. Ann Intern Med. 2010;153(10): 666-670.

17. Goldman DP, Joyce GF, Zheng Y. Prescription drug cost sharing: associations with medication and medical utilization and spending and health. JAMA. 2007;298(1):61-69.

18. DiMatteo MR. Variations in patients' adherence to medical recommendations: a quantitative review of 50 years of research. Med Care. 2004;42(3):200-209.

19. Goldman DP, Joyce GF, Escarce JJ, et al. Pharmacy benefits and the use of drugs by the chronically ill. JAMA. 2004;291(19):2344-2350.

20. Tseng CW, Tierney EF, Gerzoff RB, et al. Race/ethnicity and economic differences in cost-related medication underuse among insured adults with diabetes: the Translating Research Into Action for Diabetes Study. Diabetes Care. 2008;31(2):261-266. 